Original Research Paper

\title{
Producing Functional Fatty Acid DHA from Corn Steep Liquor by Aurantiochytrium SW1
}

\author{
${ }^{1,2}$ Xiaojie Ren, ${ }^{1}$ Qi Yan, ${ }^{1}$ Baoyue Liu, ${ }^{3}$ Yu Du, ${ }^{1,3,4,5}$ Xinhe Zhao and ${ }^{1}$ Yuanda Song \\ ${ }^{1}$ Colin Ratledge Center for Microbial Lipids, School of Agriculture Engineering and Food Science, \\ Shandong University of Technology, Zibo, China \\ ${ }^{2}$ Baolingbao Biology Co. Ltd., Dezhou, Shandong, China \\ ${ }^{3}$ Chongqing Academy of Science and Technology, Chongqing, China \\ ${ }^{4}$ State Key Laboratory of Microbial Technology, Shandong University, Qingdao, China \\ ${ }^{5}$ Luzhou Bio-Chem Technology Limited, Linyi, China
}

Article history

Received: $30-08-2021$

Revised: $13-10-2021$

Accepted: 30-10-2021

Corresponding Author:

Xinhe Zhao

School of Agriculture

Engineering and Food Science,

Colin Ratledge Center for

Microbial Lipids, China

Email: zhaoxinhe2018@163.com

\begin{abstract}
Thraustochytrium has a relatively high lipid content in the cell and its lipid content can account up to $70 \%$ of the dry cell weight. It is an important raw material for the third-generation biodiesel production and the production of functional fatty acid Docosahexaenoic Acid (DHA). However, the high cost of traditional fermentation medium has hindered the industrial production of lipid. Therefore, searching for raw materials that can be used by thraustochytrid with a wide range of sources and low prices has become a research hotspot in the production of lipids by thraustochytrid. In this study, corn steep liquor was applied for lipid production in thraustochytrid Aurantiochytrium SW1, various factors of corn medium were optimized by using Response Surface Methodology (RSM), including glucose concentration, Rotation Speed, Kinetin concentration and dilution rate of corn steep liquor. The optimal conditions were finally obtained as $72.37 \mathrm{~g} / \mathrm{L}$ glucose, $2.28 \mathrm{mg} / \mathrm{L}$ kinetin, $270 \mathrm{rpm}$ of rotation speed and 15 times of corn pulp dilution. The biomass, lipid and DHA yields obtained under the optimized conditions were $13.5,7.9$ and $42 \%$ of total fatty acids, respectively. Comparison with the traditional medium, the biomass, lipid and DHA production were promoted by $14.8,31.6$ and $31.3 \%$ respectively. The optimized medium using cheap corn steep liquor showed a better production level, which proved the feasibility of culturing Aurantiochytrium SW1 with cheap raw materials.
\end{abstract}

Keywords: Aurantiochytrium Sw1, Dha, Corn Steep Liquor, Medium Optimization, Response Surface

\section{Introduction}

Nowadays, the problems such as environmental pollution and energy crisis are becoming more and more serious in the world. New energy sources are being developed constantly. Among them, microbial fermentation to produce lipids has been a hot research topic of bio-oil production (Pinzi et al., 2014). Microbes have the characteristics of rapid growth, easy cultivation, high cell density, rich yield and are not affected by climate and site changes. It is a high-quality oil-producing raw material for sustainable cultivation (Ma, 2016). Among these high-quality bio-oils, DHA has attracted much attention as a high-quality fatty acid that cannot be synthesized by higher organisms themselves (Santigosa et al., 2021). However, with the economic development and environmental pollution, the traditional sources of DHA can no longer meet the needs of people and new sources of DHA are urgently desired. The Aurantiochytrium SW1 as a marine alga can produce a large amount of lipids, up to $50 \%$ of the biomass and DHA can account for more than $40 \%$ of the Total Fatty Acids (TFA), which is a reliable new source of DHA (Burja et al., 2006; Huang et al., 2003). With the gradual discovery of microorganisms that can produce DHA, more and more researchers have optimized the fermentation process of microorganisms, among which the continuous exploration of culture medium has been a hot research topic. However, most of the culture medium are costly and have low conversion rates, which are not conducive to the large-scale fermentation of 
microorganisms. Therefore, it is the goal of researchers to find low-cost, high-conversion rate and environmentally friendly medium raw materials.

Corn steep liquor is a by-product of agricultural products processing. Its unpleasant smell and high content of inorganic salts limit its application. The continuous accumulation of corn steep liquor has caused environmental pollution and hindered the development of crop processing enterprises Xing et al. (2010). However, corn steep liquor is rich in nutrients. With its rich nitrogen source, carbon source and a large amount of inorganic salts, it is extremely friendly to marine microorganisms and has great potential in biological fermentation (Li et al., 2013; Yokochi et al., 1998). Therefore, the development of microalgae medium by using corn steep liquor can make full use of the nutrients in corn steep liquor and relieve the pressure brought by corn steep liquor on the environment and society.

In this study, corn steep liquor was used as the medium. The influencing factors on lipid production mainly included the dilution ratio of corn steep liquor, the carbon source concentration, plant hormone concentration and the rotation speed of the shaker. Then the data were analyzed by Analysis of Variance (ANOVA) using the experimental design of the central composite design. In this way, the highest biomass, the highest lipid yield and the highest DHA content using corn steep liquor were obtained and a new idea for the utilization of corn steep liquor was also provided.

\section{Materials and Methods}

\section{Experimental Strains and Culture Media}

Aurantiochytrium SW1 was obtained from the conserved strain at the Colin Ratledge center for microbial lipids, Shandong University of Technology. Corn steep liquor was provided by Luzhou Biotechnology Co., LTD, China. The seed medium used in this experiment was: Glucose $60 \mathrm{~g} / \mathrm{L}$, yeast extract $2 \mathrm{~g} / \mathrm{L}$, sodium glutamate $8 \mathrm{~g} / \mathrm{L}$ and sea salt $6 \mathrm{~g} / \mathrm{L}$. The seed culture was incubated at $\mathrm{pH} 7.8,28^{\circ} \mathrm{C}$ and $130 \mathrm{rpm}$.

\section{Single-Factor Test to Determine the Range of Each Factor}

In this experiment, four factors, namely glucose concentration, corn steep liquor dilution rate, rotation speed and kinetin concentration, were determined as the factors affecting the growth of Aurantiochytrium SW1 and the accumulation of lipids. Firstly, the optimal parameter range of each factor is determined through a single factor experiment and then the response surface experiment is used to observe the influence of each factor on the test index. Single-factor experimental for corn steep liquor dilution rate were designed as:
Glucose $55 \mathrm{~g} / \mathrm{L}$, rotation speed 130r pm, kinetin $1.4 \mathrm{mg} / \mathrm{L}$ with $0,1,5,10,15,20$ times dilution of corn steep liquor respectively. Single-factor experimental for kinetin concentration were designed as: Glucose $55 \mathrm{~g} / \mathrm{L}, 10$ times corn steep liquor dilution, rotation speed $130 \mathrm{rpm}$ with $0,0.2$, $0.5,1,2,3,4,5 \mathrm{mg} / \mathrm{L}$ kinetin respectively. The medium for the single-factor experimental culture of Aurantiochytrium $\mathrm{SW} 1$ was $\mathrm{pH} 7.8$ at temperature $28^{\circ} \mathrm{C}$.

\section{Response Surface Ccd Experiments}

The optimal range of each factor was determined by single-factor test and four-factor CCD experiments were designed as in Table 1.

\section{Model Validation}

The optimal combination of factors was obtained based on the results of response surface analysis as shown in Table 2. The incubation temperature was $28^{\circ} \mathrm{C}$, pH was 7.8 .

\section{Determination of Biomass, Lipids and Fatty Acids}

The growth of Aurantiochytrium SW1 was measured by the absorbance of microalgal cells diluted five times at OD625 $\mathrm{nm}$. The algae cells were obtained by centrifuge algae culture at $10,000 \times \mathrm{g}$ for $10 \mathrm{~min}$ and after two times washing with ddH2O, cells were freeze-dried for $48 \mathrm{~h}$ to a constant weight. Biomass obtained was expressed as gram dried cell per liter of cultivation medium Folch et al. (1957). Lipid were extracted by chloroform/methanol (2:1) (Zhao et al., 2020) and the extracts were vaporized to a constant weight by N2. A gas chromatographic method was used to detect fatty acids profile in microalgae cells. The GC was equipped with a DB-WAX column. The temperature of the $\mathrm{GC}$ column was hold at $80^{\circ} \mathrm{C}$ for 10 minutes and then increased to $160^{\circ} \mathrm{C}$ at a rate of $8^{\circ} \mathrm{C} / \mathrm{min}$, then heated up to $220^{\circ} \mathrm{C}$ at $4^{\circ} \mathrm{C} / \mathrm{min}$. The gas flow rate was 260, 90 and $400 \mathrm{~m} / \mathrm{s}$ for He (helium), H2 (hydrogen) and air respectively; The injection volume was $1 \mu \mathrm{L}$ and the retention time was $35 \mathrm{~min}$. Design-Expert was used to process data analysis.

Table 1: The range of each factor for CCD experiment

\begin{tabular}{llllll}
\hline Name & Units & Low & High & +alpha & -alpha \\
\hline $\mathrm{A}$ & $\mathrm{g} / \mathrm{L}$ & 30 & 80 & 30 & 80 \\
$\mathrm{~B}$ & $\mathrm{mg} / \mathrm{L}$ & 0.2 & 2.6 & 0.2 & 2.6 \\
$\mathrm{C}$ & $\mathrm{rpm}$ & 130 & 270 & 130 & 270 \\
$\mathrm{D}$ & & 5 & 15 & 5 & 15 \\
\hline
\end{tabular}

Table 2: Optimal combination of factors

\begin{tabular}{lccll}
\hline Name & Glucose & Kinetin & $\begin{array}{l}\text { Rotation } \\
\text { speed }\end{array}$ & $\begin{array}{l}\text { Dilution } \\
\text { rate }\end{array}$ \\
\hline $\begin{array}{l}\text { After } \\
\text { optimization }\end{array}$ & $72.37 \mathrm{~g} / \mathrm{L}$ & $2.28 \mathrm{mg} / \mathrm{L}$ & $270 \mathrm{rpm}$ & 15 times \\
\hline
\end{tabular}




\section{Results and Discussion}

\section{Single-Factor Test Results}

In the previous research, we have tested the nutrient content of corn steep liquor and found that the corn steep liquor contains abundant amino acids and protein. The main ingredients can be seen in Table 3 Gao et al. (2013). At this stage, we studied the effect of different dilution rate of corn steep liquor on cell biomass to test whether Aurantiochytrium SW1 can live in a medium containing only corn steep liquor.

It can be seen from Fig. 1 that Aurantiochytrium SW1 grew in different dilution ratio of corn steep liquor medium. The results indicated that corn steep liquor could be used as the sole additive in the culture medium. The corn steep liquor contains abundant nitrogen sources, carbon sources and inorganic salts, which can meet the nutritional requirements of microalgae. The results show that the growth of Aurantiochytrium SW1 was high and has little difference when the dilution rate was between 10-20 times and the growth condition is the best when the concentration is diluted about 10. While in the case of raw corn steep liquor (dilution rate 0 ) or dilution rate of 1 time, only weak growth was maintained. This is because a high osmotic pressure is formed in a high concentration culture, which very easily affects the absorption of nutrients from the environment by microalgae cells and in turn causes slow growth and even death of microalgae cells. In contrast, in highly diluted corn steep liquor, the nutrient concentration is low and the nutrients within the environment are not sufficient to sustain the continued growth of microalgal cells (Gao et al., 2013). In addition, the concentration of total acid in corn steep liquor was higher at low dilution cultures, which caused a certain toxic effect on cells. Therefore, in this experiment, a dilution of 5-15 times was chosen as the range of response surface experiments in order to obtain the optimal dilution rates of corn steep liquor for the cultivation of Aurantiochytrium SW1.

In order to further improve the biomass of Aurantiochytrium SW1 in the corn steep liquor medium, we also considered other related factors. According to previous studies, carbon sources have a significant effect on the growth of microalgae. Glucose has the most obvious promoting effect on the growth of Aurantiochytrium SW1 at 50-70 g/L Ganuza and Izquierdo (2007) However, the carbon source concentration in the current 10-fold dilution of corn steep liquor medium is far from enough, so $30-80 \mathrm{~g} / \mathrm{L}$ glucose was added as the concentration range of glucose single factor for the next response surface experiment.
Different from other factors, the increase of rotation speed of the shaker has promoted the cell growth in the single factor test. However, previous studies have found that although higher shaking speed can promote the cell growth, it can inhibit the accumulation of lipid in microalgae. Studies have shown that when the rotation speed is between 200-250 rpm, the lipid content reaches the maximum. So, the range of the rotation speed 130-270 rpm was choosing to study the influence of the rotation speed on the accumulation of biomass and lipids.

In addition, phytohormones were recently discovered to stimulate the growth and biosynthesis of fatty acids in microalgae (Manikan et al., 2015; Ganuza and Izquierdo, 2007). In the previous work, we studied the effect of different phytohormones on the growth and lipid accumulation in Aurantiochytrium SW1. Among six classes of phytohormones, kinetin was the most effective hormone. Therefore, here we also tested the optimal range of kinetin concentration under constant parameters of other three factors. The results showed that the concentration of kinetin at $2 \mathrm{mg} / \mathrm{L}$ had the most obvious promoting effect on biomass.

\section{Ccd Experimental Results and Analysis}

In this study, four factors, namely glucose concentration, kinetin concentration, corn steep liquor dilution rate and rotation speed (related to dissolved oxygen), were optimized to enhance the accumulation of biomass, lipid and DHA production in Aurantiochytrium SW1.

Optimization experiments were conducted using Response Surface Design (RSM) based on Central Combinatorial Design (CCD) for Aurantiochytrium SW1 regarding four factors. Thirty sets of experiments on each combination of factors were conducted and data on biomass, lipids and DHA production were obtained for the 30 sets of experiments (Table 4).

\section{Optimization of Mficroalgal Biomass}

Using biomass as the response value, the quadratic regression equation analysis was performed on the biomass data in the Table 5. using design-expert software and the quadratic regression equation was obtained as:

$$
\begin{aligned}
& \text { Biomass }=13.41-0.31 A+0.24 \mathrm{~B}+2.70 C+0.28 D- \\
& 0.12 A B+0.32 A C+0.21 A D-0.26 B C-0.099 B D+ \\
& 0.059 C D-1.38 A^{2}-1.68 B^{2}-0.81 C^{2}-0.32 D^{2}
\end{aligned}
$$

\begin{tabular}{|c|c|c|c|c|c|c|}
\hline \multirow[b]{2}{*}{ Components } & \multicolumn{6}{|l|}{ Ingredients } \\
\hline & $\begin{array}{l}\text { Total amino } \\
\text { acids }(\mathrm{mg} / \mathrm{g})\end{array}$ & $\begin{array}{l}\text { Amino acid } \\
\text { nitrogen }(\mathrm{g} / \mathrm{L})\end{array}$ & $\begin{array}{l}\text { Reducing } \\
\text { sugar }(\mathrm{g} / \mathrm{L})\end{array}$ & $\begin{array}{l}\text { Total acid } \\
\text { (\% dry basis) }\end{array}$ & $\begin{array}{l}\text { Dissolved } \\
\text { phosphorus (g/L) }\end{array}$ & Heavy metals \\
\hline Content & 298.71 & $9.71 \pm 0.18$ & $40.33 \pm 0.92$ & $7.69 \pm 0.12$ & $4.39 \pm 1.18$ & Not Detected \\
\hline
\end{tabular}

Table 3: Main components of corn steep liquor 
Xiaojie Ren et al. / American Journal of Biochemistry and Biotechnology 2021, 17 (4): 373.383 DOI: 10.3844/ajessp.2021.373.383

Table 4: Data of each tested groups

\begin{tabular}{|c|c|c|c|c|c|c|c|}
\hline Experimental number & A & $\mathrm{B}$ & $\mathrm{C}$ & $\mathrm{D}$ & Biomass $(\mathrm{g} / \mathrm{L})$ & Lipid $(g / L)$ & DHA ( $\%$ of total fatty acids) \\
\hline 1 & 80 & 0.2 & 130 & 15 & 5.99 & 1.53 & 9.15 \\
\hline 2 & 80 & 2.6 & 130 & 5 & 5.97 & 1.45 & 19.16 \\
\hline 3 & 80 & 2.6 & 270 & 15 & 12.32 & 6.53 & 51.03 \\
\hline 4 & 30 & 0.2 & 130 & 5 & 6.62 & 1.40 & 54.06 \\
\hline 5 & 30 & 2.6 & 270 & 5 & 12.33 & 4.96 & 21.10 \\
\hline 6 & 30 & 2.6 & 130 & 15 & 8.15 & 3.35 & 35.47 \\
\hline 7 & 80 & 2.6 & 270 & 5 & 11.34 & 2.83 & 45.24 \\
\hline 8 & 55 & 1.4 & 200 & 10 & 13.83 & 6.25 & 32.44 \\
\hline 9 & 30 & 2.6 & 270 & 15 & 11.47 & 7.91 & 24.09 \\
\hline 10 & 55 & 1.4 & 200 & 10 & 13.23 & 6.45 & 32.46 \\
\hline 11 & 80 & 0.2 & 130 & 5 & 5.32 & 1.13 & 21.73 \\
\hline 12 & 55 & 2.6 & 200 & 10 & 12.09 & 5.59 & 34.88 \\
\hline 13 & 55 & 1.4 & 200 & 5 & 12.68 & 5.09 & 30.19 \\
\hline 14 & 30 & 0.2 & 130 & 15 & 6.39 & 1.52 & 28.21 \\
\hline 15 & 55 & 1.4 & 200 & 10 & 13.25 & 6.23 & 32.30 \\
\hline 16 & 55 & 1.4 & 270 & 10 & 15.32 & 7.57 & 24.83 \\
\hline 17 & 80 & 1.4 & 200 & 10 & 11.32 & 4.65 & 35.85 \\
\hline 18 & 80 & 2.6 & 130 & 15 & 6.57 & 2.33 & 38.27 \\
\hline 19 & 55 & 0.2 & 200 & 10 & 11.15 & 4.45 & 32.85 \\
\hline 20 & 55 & 1.4 & 200 & 15 & 13.28 & 8.22 & 36.28 \\
\hline 21 & 80 & 0.2 & 270 & 15 & 12.89 & 7.28 & 15.60 \\
\hline 22 & 30 & 1.4 & 200 & 10 & 12.51 & 5.17 & 32.80 \\
\hline 23 & 55 & 1.4 & 200 & 10 & 13.83 & 6.00 & 36.48 \\
\hline 24 & 55 & 1.4 & 200 & 10 & 13.43 & 5.88 & 37.09 \\
\hline 25 & 30 & 0.2 & 270 & 15 & 12.33 & 5.95 & 30.22 \\
\hline 26 & 30 & 2.6 & 130 & 5 & 7.45 & 2.14 & 30.03 \\
\hline 27 & 55 & 1.4 & 130 & 10 & 9.66 & 2.97 & 23.52 \\
\hline 28 & 30 & 0.2 & 270 & 5 & 11.39 & 2.82 & 50.43 \\
\hline 29 & 80 & 0.2 & 270 & 5 & 11.26 & 2.85 & 36.68 \\
\hline 30 & 55 & 1.4 & 200 & 10 & 13.6 & 5.80 & 36.88 \\
\hline
\end{tabular}

Table 5: Analysis of variance for Aurantiochytrium SW1 biomass

\begin{tabular}{|c|c|c|c|c|c|}
\hline Source of variance & Sum of squares & Degree of freedom & Mean Square & F-value & $\mathrm{P}$-value \\
\hline Model & 241.25 & 14 & 17.23 & 123.83 & $<0.0001$ \\
\hline $\mathrm{A}$ & 1.78 & 1 & 1.78 & 12.79 & 0.0028 \\
\hline B & 1.05 & 1 & 1.05 & 7.55 & 0.0149 \\
\hline $\mathrm{C}$ & 130.84 & 1 & 130.84 & 940.22 & $<0.0001$ \\
\hline $\mathrm{D}$ & 1.41 & 1 & 1.41 & 10.10 & 0.0062 \\
\hline $\mathrm{AB}$ & 0.23 & 1 & 0.23 & 1.67 & 0.2154 \\
\hline $\mathrm{AC}$ & 1.59 & 1 & 1.59 & 11.45 & 0.0041 \\
\hline $\mathrm{AD}$ & 0.69 & 1 & 0.69 & 4.98 & 0.0413 \\
\hline $\mathrm{BC}$ & 1.12 & 1 & 1.12 & 8.04 & 0.0125 \\
\hline $\mathrm{BD}$ & 0.16 & 1 & 0.16 & 1.14 & 0.3035 \\
\hline $\mathrm{CD}$ & 0.056 & 1 & 0.056 & 0.41 & 0.5339 \\
\hline $\mathrm{A} 2$ & 4.96 & 1 & 4.96 & 35.66 & $<0.0001$ \\
\hline B2 & 7.30 & 1 & 7.30 & 52.49 & $<0.0001$ \\
\hline $\mathrm{C} 2$ & 1.70 & 1 & 1.70 & 12.19 & 0.0033 \\
\hline D2 & 0.26 & 1 & 0.26 & 1.90 & 0.1888 \\
\hline Residual & 2.09 & 15 & 0.14 & & \\
\hline Lack of fit & 1.72 & 10 & 0.17 & 2.37 & 0.1762 \\
\hline Pure error & 0.36 & 5 & 0.073 & & \\
\hline Cor total & 243.34 & & & & \\
\hline Std.Dev. & 0.37 & & $\mathrm{R}^{2}$ & 0.9914 & \\
\hline Adeq precision & 39.113 & & Adj $R^{2}$ & 0.9834 & \\
\hline
\end{tabular}


We performed variance analysis on the quadratic model and the results are shown in Table 5. It can be seen that the $\mathrm{p}$-value of the lack of fit was 0.1762 , ( $\mathrm{p}>0.05$, not significant) and the square of the model correlation coefficient $\mathrm{R}^{2}=0.9914$, which indicated that the model is extremely significant. In this model, the effects of glucose, rotation speed, kinetin concentration and dilution rate of corn pulp on the accumulation of biomass were significant. This indicates that the variability of experimental data can be explained by this model, the confidence level of the model is high, the fitness is good and the equation can reflect the real experimental values.

Therefore, we use this equation to analyze and predict the experimental results. The effect of the interaction of A, B, C and D on biomass is shown in Fig. 2.

The experimental results revealed that the accumulation of Aurantiochytrium SW1 was the highest $(15.32 \mathrm{~g} / \mathrm{L})$ when the rotation speed was $270 \mathrm{rpm}$, the kinetin concentration was $1.4 \mathrm{mg} / \mathrm{L}$, the glucose concentration was $55 \mathrm{~g} / \mathrm{L}$ and the dilution rate of corn pulp 10 times. The effect of rotation speed, i.e., the amount of dissolved oxygen in the medium, on the accumulation of biomass was the most significant. When the rotation speed was $130 \mathrm{rpm}$, the biomass of Aurantiochytrium.SW1 was all below $10 \mathrm{~g} / \mathrm{L}$ and when the rotation speed was $270 \mathrm{rpm}$, the biomass of Aurantiochytrium SW1 was all at a higher level $(11.26-15.32 \mathrm{~g} / \mathrm{L})$. This was due to the fact when the oxygen in the medium was insufficient, it was difficult to sustain the growth of Aurantiochytrium SW1.This is consistent with the study of Zhang et al. (2018), which also showed that oxygen is an important factor for the growth of Aurantiochytrium SW1. It was found that the addition of a moderate amount of glucose also contributed to the growth of Aurantiochytrium SW1 at lower rotational speeds. In group 27, the microalgae grew well at low rotational speed (130 rpm), obtaining biomass close to $10 \mathrm{~g} / \mathrm{L}$, indicating that the carbon source is also an important factor for the growth of microalgae. In the analysis of the interaction between the two factors, it was also found that their $\mathrm{R}$ value was much lower than 0.05 , reflecting a high significance.

In the analysis of the independent effect of glucose on biomass accumulation, it was found that the optimal concentration to promote biomass accumulation was $59 \mathrm{~g} / \mathrm{L}$. Glucose concentrations above this value negatively affected the growth of Aurantiochytrium SW1. After an in-depth analysis of the corn steep liquor composition, it was found that with the addition of the sugars contained in the corn steep liquor, the medium reached a sugar concentration of $70 \mathrm{~g} / \mathrm{L}$. This result is consistent with the studies of Nazir et al. $(2020 ; 2018)$, who showed that the optimal carbon source concentration for the growth and DHA production of Aurantiochytrium SW1 is in the range of $60-100 \mathrm{~g} / \mathrm{L}$ and the growth of Aurantiochytrium SW1 is inhibited with carbon sources above $100 \mathrm{~g} / \mathrm{L}$.

The study also showed that the interaction of rotation speed and kinetin addition also exhibited a high significance on biomass accumulation. Kinetin, also known as cytokinin, is good at promoting cell division in the appropriate concentration range. However, high concentrations of kinetin can inhibit cell division. In this study, we found that kinetin concentration around $1.25 \mathrm{mg} / \mathrm{L}$ can promote cell production well. Above this value, kinetin has a negative impact on Aurantiochytrium SW1 biomass accumulation.

In the experiment, it was found that $\mathrm{C} / \mathrm{N}$ ratio (glucose concentration/dilution rate of corn steep liquor) has an important influence on Aurantiochytrium SW1 biomass accumulation. The accumulation of biomass was least when both factors were at high levels. Even at high rotation speed, Aurantiochytrium SW1 was still unable to break the negative effect of high concentration of glucose and nitrogen source. This may because the high osmotic pressure of the medium has a greater effect on the cells and the high osmotic pressure leads to the loss of cytolymph and a smaller cell size, which can only maintain the basic physiological activities Chen et al. (2010). However, the lower concentrations of carbon and nitrogen sources were not sufficient to maintain the continuous growth of the cells, resulting in cell starvation and inability to reach higher biomass levels. These results are consistent with previous studies (Furlan et al., 2017; Li et al., 2009; Hong et al., 2013). where high concentrations of nitrogen inhibited the growth of Aurantiochytrium SW1. There is also the possibility that the change in $\mathrm{pH}$ in the medium caused by the microorganism when using nitrogen source which affect the growth of cells.

\section{Optimization of Lipid Production by Microalgae}

Using lipid content as the response value, the quadratic regression equation for lipid was obtained from Table 6 using Design-Expert software:

$$
\begin{aligned}
& \text { Lipid }=6.11-0.26 A+0.45 B+1.72 C+1.11 D- \\
& 0.40 A B-0.012 A C+0.12 A D-0.023 B C+0.041 B D \\
& +0.73 C D-1.21 A^{2}-1.10 B^{2}-0.85 C^{2}+0.54 D^{2}
\end{aligned}
$$

The results of variance analysis on the quadratic model are shown in Table 6 . It shows that the p-value of the misfit term test was $0.0558,(\mathrm{p}>0.05$, not significant) and the correlation coefficient $R^{2}=09778$, which suggested the model was significant. In this model, the effects of glucose concentration, rotation speed, kinetin concentration and dilution rate of corn steep liquor were significant on the accumulation of lipids. It shows that the variability of experimental data can be explained by this model and the model has high confidence and good fitting degree and the equation can reflect the real experimental value better.

Therefore, this equation can be used to analyze and predict the experimental results. Using the Design-Expert software to make a three-dimensional diagram of each factor and the effects of A, B, C and D and their interactions on the response surface were shown in Fig. 3. 
Table 6: Analysis of variance for Aurantiochytrium SW1 lipid accumulation

\begin{tabular}{llllll}
\hline Source of variance & Sum of square & Degree of freedom & Mean Square & F-value & P-value \\
\hline Model & 133.93 & 14 & 9.57 & 47.21 & $<0.0001$ \\
A & 1.21 & 1 & 1.21 & 5.96 & 0.0275 \\
B & 3.70 & 1 & 3.70 & 261.47 & 0.0007 \\
C & 52.98 & 1 & 52.98 & 109.09 & $<0.0001$ \\
D & 22.10 & 1 & 22.10 & 12.40 & $<0.0001$ \\
AB & 2.51 & 1 & 2.51 & 0.012 & 0.0031 \\
AC & $2.351 \mathrm{E}-003$ & 1 & $2.351 \mathrm{E}-003$ & 1.20 & 0.9156 \\
AD & 0.24 & 1 & 0.24 & 0.041 & 0.2906 \\
BC & $8.376 \mathrm{E}-003$ & 1 & $8.376 \mathrm{E}-003$ & 0.13 & 0.8416 \\
BD & 0.027 & 1 & 0.027 & 41.57 & 0.7225 \\
CD & 8.42 & 1 & 8.42 & 18.67 & $<0.0001$ \\
A2 & 3.78 & 1 & 3.78 & 15.33 & 0.0006 \\
B2 & 3.11 & 1 & 3.11 & 9.16 & 0.0014 \\
C2 & 1.86 & 1 & 1.86 & 3.74 & 0.0085 \\
D2 & 0.76 & 15 & 0.76 & 4.48 & 0.0722 \\
Residual & 3.04 & 10 & 0.20 & 0.27 & 0.0558 \\
Lack of Fit & 2.73 & 5 & 0.061 & & \\
Pure Error & 0.31 & 29 & $\mathrm{R}^{2}$ & Adj R & 0.9778 \\
Cor Total & 136.97 & & & 0.9571 & \\
Std.Dev. & 0.45 & & & & \\
Adeq Precision & 22.385 & & & &
\end{tabular}
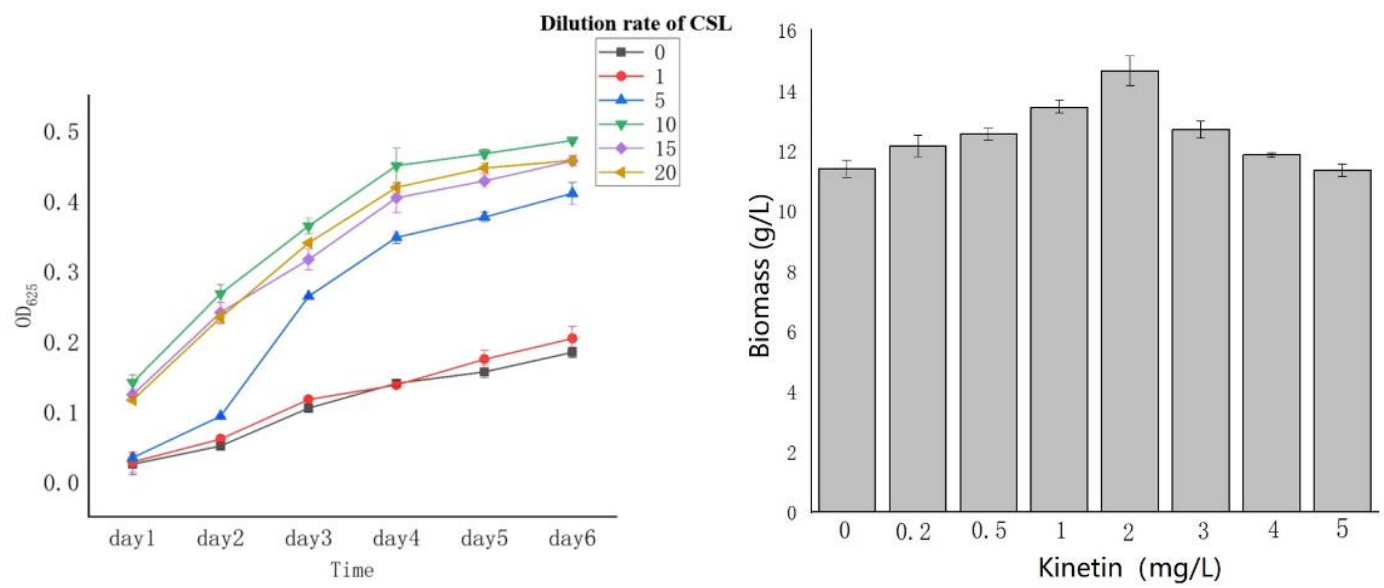

Fig. 1: Effect of factors on biomass accumulation $\left(O D_{625}\right.$ represents the absorbance of the Aurantiochytrium SW1culture; $0,1,5,10,15,20$ represent different dilution rates of the corn steep liquor; Biomass represents the Aurantiochytrium SW1 dry weight collected from per liter of different cultures. Kinetin represents the concentration of plant hormone added in different cultures)
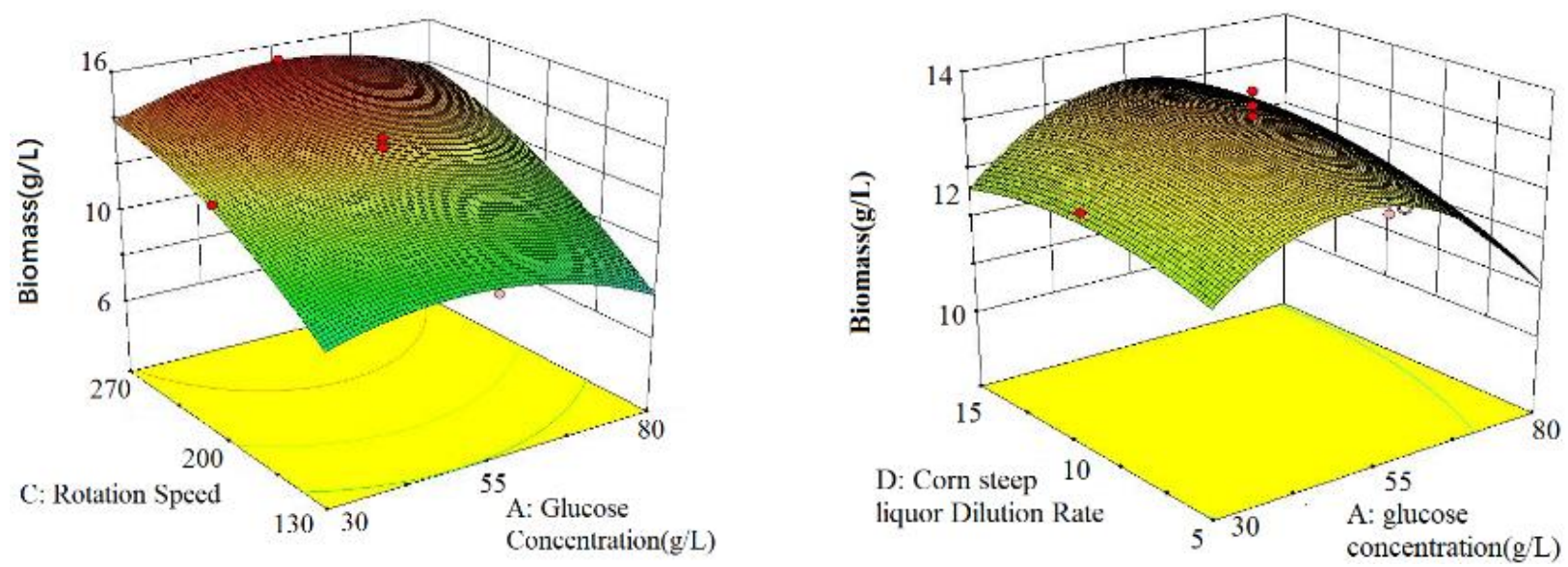


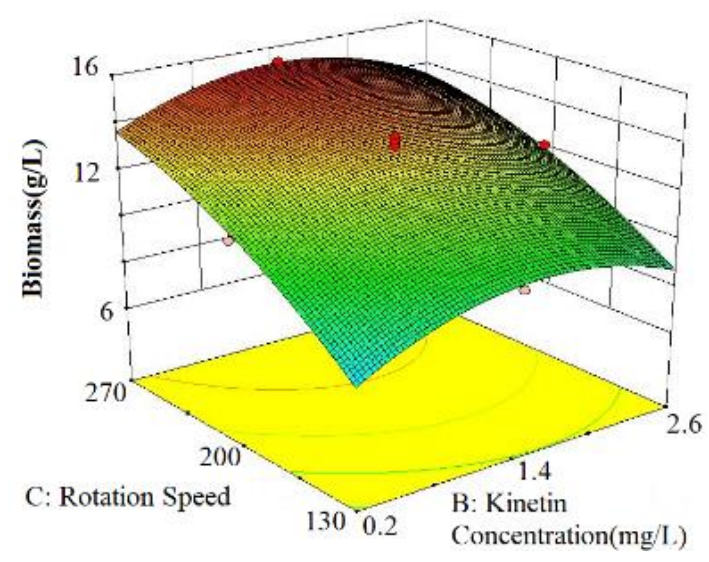

Fig. 2: Influence of factor interaction on biomass (The ordinate represents the index to be optimized, which is biomass, the dry weight of Aurantiochytrium SW1 obtained per liter of culture solution under different combinations of culture factors. These culture factors are reflected in the abscissa, including rotation speed of the shaker, glucose concentration in the culture, corn steep liquor dilution rate and the plant hormone, kinetin concentration in the culture)
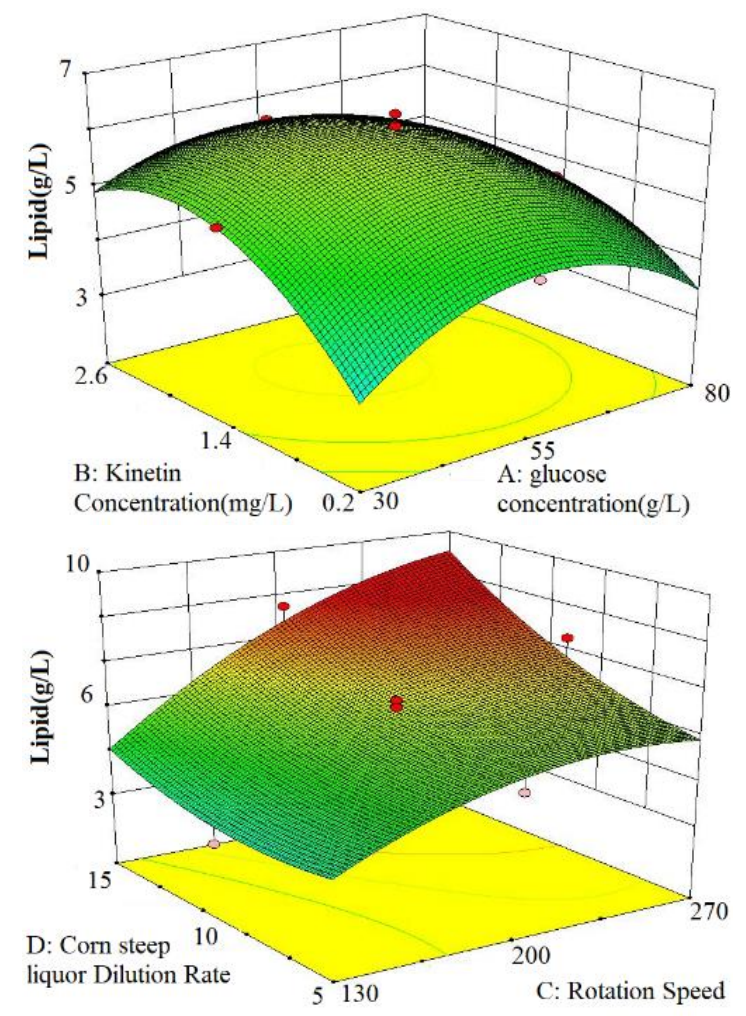

Fig. 3: Influence of factor interaction on lipid accumulation (The ordinate represents the index to be optimized, which is the weight of lipid obtained per liter of Aurantiochytrium SW1 culture under different combinations of culture factors. These culture factors are reflected in the abscissa, including rotation speed of the shaker, glucose concentration in the culture, corn steep liquor dilution rate and the plant hormone, kinetin concentration in the culture)
It was found from the CCD experiments that when the glucose was about $55 \mathrm{~g} / \mathrm{L}$, the rotation speed was $200 \mathrm{rpm}$ and the dilution ratio of corn pulp was 15 times, the lipid accumulation reached highest of $8.2 \mathrm{~g} / \mathrm{L}$. Obviously, the accumulation of a large number of cells has a great impact on the accumulation of lipid. Since the amount of lipid accumulation of microalgae is closely related to the growth of microalgae cells, therefore, the factors favorable to the growth of microalgae cells are also favorable to the lipid production rate of cells (Wong et al., 2008; Aasen et al., 2016) However, the experimental combination with the highest lipid accumulation was no longer the one with the highest biomass accumulation, but the one with a glucose concentration of $55 \mathrm{~g} / \mathrm{L}$ and the dilution ratio of corn pulp was 15 times. This result may have occurred because the carbon to nitrogen ratio in the medium affected the lipid accumulation of microalgae. According to previous studies 9 (Li et al., 2015) when carbon and nitrogen sources are consumed in a certain proportion, microalgae will start to use carbon sources in the medium to accumulate lipid. Here we found that the best experimental combination of carbon and nitrogen ratio was about 30. However, under the combination of high concentration of glucose and corn pulp, lipid accumulation was lower and even high rotational speed could not compensate for this negative effect. This demonstrated the effect of high osmotic pressure on cell biomass accumulation in the previous section, thus reducing lipid production.

From Table 6 it can be seen that the rotation speed continues to show a greater effect on the accumulation of lipids. It has been claimed Song et al. (2007) that higher rotation speed is necessary to obtain a higher biomass, but appropriate rotation speed is beneficial for the accumulation of lipids in microalgae. There are two main pathways of lipid synthesis in microalgae, the FAS pathway requires a large amount of oxygen, but PKS does not require the participation of oxygen, which suggests that the accumulation of lipids in Aurantiochytrium SW1 has a high demand for oxygen. Besides this, in the analysis of lipid fatty acids, it was found that Aurantiochytrium SW1 produced C15 when using this carbon and nitrogen source, indicating that the lipid accumulation pathways of Aurantiochytrium SW1 are not limited to these two lipid accumulation pathways. Because the FAS and PKS pathways are not able to accumulate singular carbon fatty acids Goncalves et al. (2016). It has been reported that Aurantiochytrium SW1 has different oxygen requirements and fatty acid accumulation pathways when using different substrates, our study indicates that Aurantiochytrium SW1 has higher oxygen requirements and different lipid accumulation pathways when using glucose as carbon source and corn pulp as nitrogen source.

\section{Optimization of Dha Production from Microalgae}

Using the DHA content as the response value, the quadratic regression equation analysis was performed on the DHA data in the Table 7. using Design-Expert software and the quadratic regression equation was obtained as: 


$$
\begin{aligned}
& D H A=33.19-1.87 A+1.13 B+2.20 C-2.24 D+ \\
& 7.67 A B+5.14 A C+1.80 A D-0.079 B C+7.07 B D- \\
& 1.16 C D+2.56 A^{2}+2.10 B^{2}-7.59 C^{2}+1.47 D^{2}
\end{aligned}
$$

The results of variance analysis on the quadratic model are shown in Table 7 . It shows that the p-value of the misfit term test was $0.0594,(\mathrm{p}>0.05$, not significant) and the model correlation coefficient $\left(\mathrm{R}^{2}=0.9029\right)$ was significant, in which the effects of glucose concentration, rotation speed and dilution rates of corn pulp on the accumulation of DHA were significant. It indicates that the variability of experimental data can be explained by this model, the confidence level of the model is high, the goodness of fit is good and the equation can better reflect the real experimental values.

Therefore, this equation can be used to analyze and predict the experimental results. Using the Design-Expert software to make a three-dimensional diagram of each factor, the effects of A, B, C, D and their interactions on DHA production are shown in Fig. 4.

As shown in Fig. 4 part of the graph at this stage is a concave surface, indicating that some of the factors had an effect on the DHA content at the center point, it can be seen that the dilution times of the corn steep liquor and the rotation speed had a significant effect on the percentage of DHA, which indicates that the adequacy of oxygen effectively affected the accumulation of DHA as well as the oil and the concentration of the nitrogen source was also an important factor affecting the synthesis of DHA.

In the one-way analysis of the rotation speed, it was found that its promotion effect on DHA reached maximum around $200 \mathrm{rpm}$. Exceeding this value will reduce the DHA production of SW1, which is probably due to the inhibition of DHA synthesis. Because increasing the rotating speed of the shaker may increase dissolved oxygen in the medium and affect the synthesis pathway of DHA. This indicates that although SW1 requires a large amount of oxygen for lipid production, it requires less oxygen during DHA production, which is consistent with the study of Goncalves et al. (2016).

As shown in Fig. 4, part of the graphics at this stage are concave curved surfaces, indicating that some factors inhibit the accumulation of DHA at the central point. Among them, the effects of kinetin and carbon source concentrations on DHA are highly significant.

The figure shows that both high and low concentrations of kinetin and carbon sources have a positive effect on the accumulation of DHA, while the central point shows an inhibitory effect on DHA. This phenomenon is exactly the opposite of the response surface results of biomass, that is, too high or too low concentrations of kinetin and carbon sources are not conducive to biomass accumulation, while appropriate concentrations of kinetin and carbon sources can promote biomass accumulation. The reason for this phenomenon may be that the above two environments are not conducive to the accumulation of biomass, so that the carbon source metabolism flows to the accumulation of lipid in the microalgae, leading to the accumulation of DHA. This is also consistent with the results of previous studies, that is, in the process of microalgae cultivation, there is a general "water-bed effect" in which biomass and lipid content fluctuate one after the other. That is to say, microalgae can reproduce quickly and accumulate biomass in a nutrient-balanced medium, but generally do not accumulate lipid; and they can only synthesize and accumulate lipid when there is a lack of nitrogen in the medium and growth is restricted (Song et al., 2007)

Interestingly, the carbon-nitrogen ratio is extremely significant in the influencing factors of lipid accumulation, but it is not highly significant in the synthesis of DHA. It can be seen from Table 7 that the dilution ratio of corn steep liquor has a significant effect on the accumulation of DHA, which shows that the concentration of nitrogen source is an important factor affecting the synthesis of DHA. This experiment shows that low-concentration corn steep liquor (diluted 15 times corn steep liquor) is not conducive to DHA synthesis. In addition, it is found from Table 7 that the rotation speed of the shaker, that is, whether the oxygen is sufficient or not, effectively affects the accumulation of DHA and lipid, which further proves that the lipid accumulation pathway of microalgae is an aerobic process.

Finally, the regression analysis based on the model equations of biomass, lipid content and DHA yield of Aurantiochytrium SW1 was performed to obtain the optimal levels of each factor. It was predicted that under $72.37 \mathrm{~g} / \mathrm{L}$ glucose, $2.28 \mathrm{mg} / \mathrm{L}$ kinetin, the shaker speed of $270 \mathrm{rpm}$ and the corn pulp dilution rate of 15 times, the optimal biomass, lipid and DHA yields will be obtained as $13.7 \mathrm{~g} / \mathrm{L}, 8.2 \mathrm{~g} / \mathrm{L}$ and $44.5 \%$ respectively.

\section{Model Validation}

Further experiments were conducted to validate the scientific validity of the model under the best estimation conditions generated by the software. The biomass, lipid and DHA yields obtained under the optimized conditions were $13.5 \mathrm{~g} / \mathrm{L}, 7.9 \mathrm{~g} / \mathrm{L}$ and $42 \%$, respectively. The values were comparable to the predicted values, which indicated that the model was true and reliable, this also demonstrated the feasibility of culturing Aurantiochytrium SW1 in corn steep liquor and the scientific validity of RSM-CCD for optimizing Aurantiochytrium SW1 culture medium.

Finally, we also compared the pre-optimized basal medium with the optimized corn medium and found the biomass, lipid and DHA production were promoted by $14.8,31.6$ and $31.3 \%$ respectively after the optimization (Table 8). This indicated that the optimized medium using cheap corn steep liquor had a better level of production, which provides a theoretical basis for the cultivation of Aurantiochytrium SW1 with corn steep liquor. 
Table 7: Analysis of variance for Aurantiochytrium SW1 DHA production

\begin{tabular}{llllll}
\hline Source of variance & Sum of square & Degree of freedom & Mean Square & F-value & P-value \\
\hline Model & 2652.97 & 14 & 189.50 & 9.96 & 3.32 \\
A & 63.08 & 1 & 63.08 & 1.21 & 0.00886 \\
B & 22.98 & 1 & 22.98 & 4.59 & 0.2890 \\
C & 87.28 & 1 & 87.28 & 4.74 & 0.0490 \\
D & 90.18 & 1 & 90.18 & 49.54 & 0.0458 \\
AB & 942.07 & 1 & 942.07 & 22.19 & $<0.0001$ \\
AC & 422.08 & 1 & 422.08 & 2.74 & 0.0003 \\
AD & 52.06 & 1 & 52.06 & $5.191 \mathrm{E}-003$ & 0.1188 \\
BC & 0.099 & 1 & 0.099 & 42.00 & 0.9435 \\
BD & 798.82 & 1 & 798.82 & 1.14 & 0.0001 \\
CD & 21.69 & 1 & 21.69 & 0.89 & 0.3024 \\
A2 & 17.02 & 1 & 17.02 & 0.60 & 0.3592 \\
B2 & 11.44 & 1 & 11.44 & 7.85 & 0.4500 \\
C2 & 149.32 & 1 & 149.32 & 0.29 & 0.0134 \\
D2 & 5.58 & 1 & 5.58 & 19.02 & 0.5960 \\
Residual & 285.27 & 15 & 25.58 & 4.34 & 0.0594 \\
Lack of Fit & 255.80 & 10 & 5.89 & &
\end{tabular}

Table 8: Compare the production ability of Aurantiochytrium SW1 in the pre-optimized and optimized medium

\begin{tabular}{llll}
\hline Indicators & Unit & Before optimization & After optimization \\
\hline Biomass & $\mathrm{g} / \mathrm{L}$ & 11.6 & 13.5 \\
Lipid yield & $\mathrm{g} / \mathrm{L}$ & 6.0 & 7.9 \\
DHA yield & & $32 \%$ & $42 \%$ \\
\hline
\end{tabular}

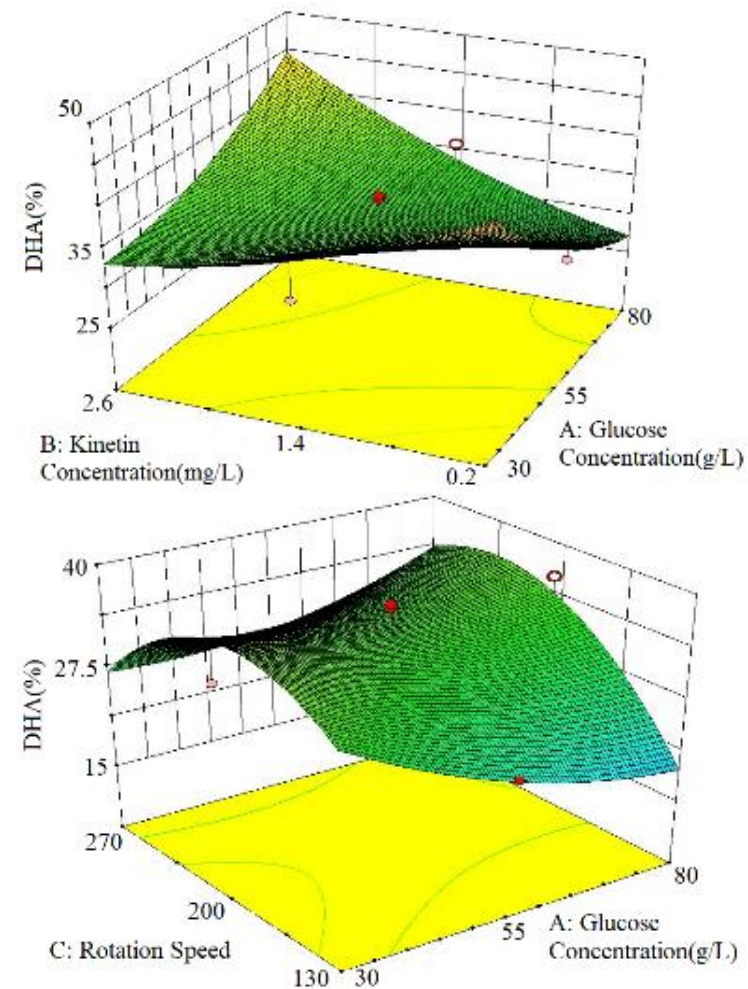

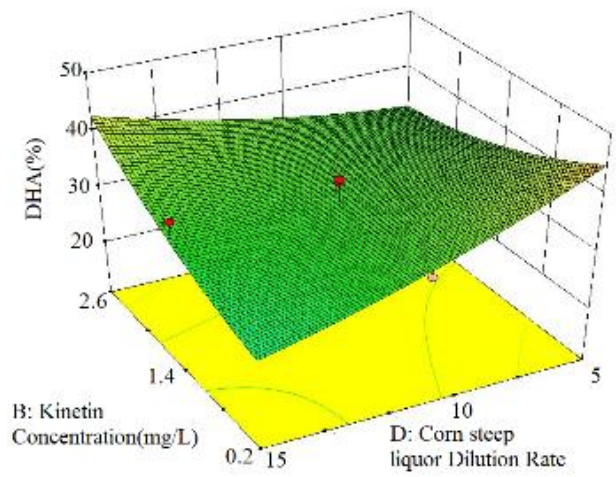

Fig. 4: Influence of factor interaction on DHA accumulation (The ordinate represents the index to be optimized, which is the DHA ratio in the total fatty acids under different combinations of culture factors. These culture factors are reflected in the abscissa, including rotation speed of the shaker, glucose concentration in the culture, corn steep liquor dilution rate and the plant hormone, kinetin concentration in the culture)

\section{Conclusion}

In this experiment, the response range of each CCD factor was determined by single-factor test. Thirty 
groups of CCD experiments were conducted by Design-Expert. The experimental factors that greatly affected biomass, lipid and DHA production were discussed. It was predicted by the model that under $72.37 \mathrm{~g} / \mathrm{L}$ glucose, $2.28 \mathrm{mg} / \mathrm{L}$ kinetin, the shaker speed of $270 \mathrm{rpm}$ and the corn pulp dilution rate of 15 times, the optimal biomass, lipid and DHA yields will be obtained as $13.7,8.2 \mathrm{~g} / \mathrm{L}$ and $44.5 \%$ respectively. In the third stage of model verification, these values were $13.5,7.9 \mathrm{~g} / \mathrm{L}$ and $42 \%$, respectively. It is consistent with the predicted value, indicating that the model is true and reliable.

It was also found that the rotation speed of the shaker was the most significant factor affecting the indexes and the accumulation of biomass was extremely low when the rotation speed was low, indicating that oxygen plays a vital role in the growth of Aurantiochytrium SW1. It was also found that the most significant factors affecting the accumulation of lipids in Aurantiochytrium SW1 were the glucose concentration and the corn pulp dilution ratio, which proved that the carbon to nitrogen ratio plays an important role in the accumulation of lipids in Aurantiochytrium. SW1. The optimized corn steep liquor medium showed better production promotion than the traditional medium, indicating the feasibility for application of cheap corn steep liquor in lipid production with Aurantiochytrium SW1.

\section{Acknowledgement}

The authors are grateful to the funding support from the Shandong Provincial Natural Science Foundation, China (ZR2019BC099 and ZR2020MC201). Chongqing technological innovation and application development key projects (cstc2019jscx-gksbX0113). China Post-Doctoral Science Foundation (2019M650167 and 2019M662362).

\section{Author's Contributions}

Xiaojie Ren and Qi Yan: Participated in the whole experiment process and also contributed to the interpretation of the results and manuscript preparation.

Baoyue Liu and Yu Du: Participated in manuscript preparation.

Xinhe Zhao and Yuanda Song: Ameliorated the manuscript and contributed to the funding support.

\section{Ethics}

This article is original and contains unpublished material. The corresponding author confirms that all of the other authors have read and approved the manuscript and no ethical issues involved.

\section{References}

Aasen, I. M., Ertesvåg, H., Heggeset, T. M. B., Liu, B., Brautaset, T., Vadstein, O., \& Ellingsen, T. E. (2016). Thraustochytrids as production organisms for docosahexaenoic acid (DHA), squalene and carotenoids. Applied microbiology and biotechnology, 100(10), 4309-4321.

https://link.springer.com/article/10.1007/s00253-0167498-4

Burja, A. M., Radianingtyas, H., Windust, A., \& Barrow, C. J. (2006). Isolation and characterization of polyunsaturated fatty acid producing Thraustochytrium species: screening of strains and optimization of omega-3 production. Applied microbiology and biotechnology, 72(6), 1161-1169. https://link.springer.com/article/10.1007/s00253006-0419-1

Chen, G., Fan, K. W., Lu, F. P., Li, Q., Aki, T., Chen, F., \& Jiang, Y. (2010). Optimization of nitrogen source for enhanced production of squalene from thraustochytrid Aurantiochytrium sp. New biotechnology, 27(4), 382-389. doi.org/10.1016/j.nbt.2010.04.005

Santigosa, E., Brambilla, F., Milanese, L. (2021) Microalgae oil as an effective alternative source of EPA and DHA for gilthead seabream (Sparus aurata) Aquaculture. Animals (Basel) 11(4): 971. doi.org/10.3390/ani11040971.

Folch, J., Lees, M., \& Stanley, G. S. (1957). A simple method for the isolation and purification of total lipides from animal tissues. Journal of biological chemistry, 226(1), 497-509.

hattps://www.jbc.org/content/226/1/497.full.pdf

Furlan, V. J. M., Maus, V., Batista, I., \& Bandarra, N. M. (2017). Production of docosahexaenoic acid by Aurantiochytrium sp. ATCC PRA-276. brazilian journal of microbiology, 48, 359-365. https://www.scielo.br/j/bjm/a/FmpSp7czfPLXFFdX cwcRkbJ/?lang=en\&format=html

Ganuza, E., \& Izquierdo, M. S. (2007). Lipid accumulation in Schizochytrium G13/2S produced in continuous culture. Applied microbiology and biotechnology, 76(5), 985-990.

https://link.springer.com/article/10.1007/s00253007-1019-4

Gao, M., Song, X., Feng, Y., Li, W., \& Cui, Q. (2013). Isolation and characterization of Aurantiochytrium species: High Docosahexaenoic Acid (DHA) production by the newly isolated microalga, Aurantiochytrium sp. SD116. Journal of oleo science, 62(3), 143-151.

https://www.jstage.jst.go.jp/article/jos/62/3/62_143/_ar ticle/-char/ja/ 
Goncalves, E. C., Wilkie, A. C., Kirst, M., \& Rathinasabapathi, B. (2016). Metabolic regulation of triacylglycerol accumulation in the green algae: Identification of potential targets for engineering to improve oil yield. Plant biotechnology journal, 14(8), 1649-1660.

https://onlinelibrary.wiley.com/doi/full/10.1111/pbi. 12523

Hong, W. K., Yu, A., Oh, B. R., Park, J. M., Kim, C. H., Sohn, J. H., ... \& Seo, J. W. (2013). Large-scale production of microalgal lipids containing high levels of docosahexaenoic acid upon fermentation of Aurantiochytrium sp. $\quad$ KRS101. https://www.scirp.org/html/1-2700719_36090.htm

Huang, J., Aki, T., Yokochi, T., Nakahara, T., Honda, D., Kawamoto, S., ... \& Suzuki, O. (2003). Grouping newly isolated docosahexaenoic acid-producing thraustochytrids based on their polyunsaturated fatty acid profiles and comparative analysis of $18 \mathrm{~S}$ rRNA genes. Marine biotechnology, 5(5), 450-457. https://link.springer.com/article/10.1007/s10126002-0110-1

Li, J., Liu, R., Chang, G., Li, X., Chang, M., Liu, Y., ... \& Wang, X. (2015). A strategy for the highly efficient production of docosahexaenoic acid by Aurantiochytrium limacinum SR21 using glucose and glycerol as the mixed carbon sources. Bioresource technology, 177, 51-57. doi.org/10.1016/j.biortech.2014.11.046.

Li, J., Liu, Y., \& Ma, J. (2013). Perspectives in DHA production of Thraustochytrids. Shipin Gongye Keji/Transactions of the Chinese Food Industry Technology. 16, 328-332. doi.org/10.13386/j.issn1002-0306.2013.16.002

Li, Q., Chen, G. Q., Fan, K. W., Lu, F. P., Aki, T., \& Jiang, Y. (2009). Screening and characterization of squalene-producing thraustochytrids from Hong Kong mangroves. Journal of agricultural and food chemistry, 57(10), 4267-4272.

https://pubs.acs.org/doi/abs/10.1021/jf9003972

Manikan, V., Nazir, M. Y. M., Kalil, M. S., Isa, M. H. M., Kader, A. J. A., Yusoff, W. M. W., \& Hamid, A. A. (2015). A new strain of docosahexaenoic acid producing microalga from Malaysian coastal waters. Algal Research, 9, 40-47. doi.org/10.1016/j.algal.2015.02.023

Nazir, Y., Halim, H., Al-Shorgani, N. K. N., Manikan, V., Hamid, A. A., \& Song, Y. (2020). Efficient conversion of extracts from low-cost, rejected fruits for high-valued Docosahexaenoic acid production by Aurantiochytrium sp. SW1. Algal Research, 50, 101977. doi.org/10.1016/j.algal.2020.101977
Nazir, Y., Shuib, S., Kalil, M. S., Song, Y., \& Hamid, A. A. (2018). Optimization of culture conditions for enhanced growth, lipid and docosahexaenoic acid (DHA) production of Aurantiochytrium SW1 by response surface methodology. Scientific reports, 8(1), 1-12. https://www.nature.com/articles/s41598018-27309-0

Pinzi, S., Leiva, D., López-García, I., Redel - Macías, M. D., \& Dorado, M. P. (2014). Latest trends in feedstocks for biodiesel production. Biofuels, Bioproducts and Biorefining, 8(1), 126-143.

Song, X., Zhang, X., Kuang, C., Zhu, L., \& Guo, N. (2007). Optimization of fermentation parameters for the biomass and DHA production of Schizochytrium limacinum OUC88 using response surface methodology. Process Biochemistry, 42(10), 1391-1397. doi.org/10.1016/j.procbio.2007.07.014

Wong, M. K., Tsui, C. K., Au, D. W., \& Vrijmoed, L. L. (2008). Docosahexaenoic acid production and ultrastructure of the thraustochytrid Aurantiochytrium mangrovei MP2 under high glucose concentrations. Mycoscience, 49(4), 266-270. doi.org/10.1007/S10267-008-0415-7

Xing, Y., Xu, L., Han, J., Xu, X., Ding, Y., Zhou, Z., \& Xu, F. (2010). Advancement of the high-value process of maize. Zhongguo Shiwu yu Yingyang/Transactions of the Chinese Food and Nutrition in China. 7, 32-35. doi.org/10.3969/j.issn.1006-9577.2010.07.009

Ma, Y., (2016). Microbial oils and its research advance. Chinese journal of bioprocess engineering, 4. https://en.cnki.com.cn/Article_en/CJFDTotalSWJG200604001.htm

Yokochi, T., Honda, D., Higashihara, T., \& Nakahara, T. (1998). Optimization of docosahexaenoic acid production by Schizochytrium limacinum SR21. Applied Microbiology and Biotechnology, 49(1), 72-76. https://link.springer.com/article/10.1007/s00253005 1139

Zhang, M., Wu, W., Guo, X., Weichen, Y., Qi, F., Jiang, X., \& Huang, J. (2018). Mathematical modeling of fed-batch fermentation of Schizochytrium sp. FJU512 growth and DHA production using a shift control strategy. 3 Biotech, 8(3), 1-7.

https://link.springer.com/article/10.1007/s13205018-1187-1.

Zhao, X., He, Z., Zhao, Y., Ma, C., \& Ren, X. (2020). Kinetic simulation of amino acid metabolism of butanol produced from the fermentation of corn steep liquor. Nongye Gongcheng Xuebao/Transactions of the Chinese Society of Agricultural Engineering 36(18),263-274 doi.org/10.11975/j.issn.1002-6819.2020.18.031. 